\title{
Novel design method for generalized lattice filters
}

\author{
Agger, Søren; Povlsen, Jørn Hedegaard; Leick, Lasse; Zauner, Dan Anker
}

Published in:

Optical Fiber Communications Conference, 2003. OFC 2003

Link to article, DOI:

10.1109/OFC.2003.1248516

Publication date:

2003

Document Version

Publisher's PDF, also known as Version of record

Link back to DTU Orbit

Citation (APA):

Agger, S., Povlsen, J. H., Leick, L., \& Zauner, D. A. (2003). Novel design method for generalized lattice filters. In Optical Fiber Communications Conference, 2003. OFC 2003 (Vol. 2, pp. 708-710). IEEE.

https://doi.org/10.1109/OFC.2003.1248516

\section{General rights}

Copyright and moral rights for the publications made accessible in the public portal are retained by the authors and/or other copyright owners and it is a condition of accessing publications that users recognise and abide by the legal requirements associated with these rights.

- Users may download and print one copy of any publication from the public portal for the purpose of private study or research.

- You may not further distribute the material or use it for any profit-making activity or commercial gain

- You may freely distribute the URL identifying the publication in the public portal

If you believe that this document breaches copyright please contact us providing details, and we will remove access to the work immediately and investigate your claim. 
3. Equivalent Noise Caused by the Inband Crosstalk

Neglecting the possible delay arising at each optical switch, a packet experiences a delay of $d_{s} \tau$ $\left(0 \leq d_{s} \leq 2^{n}-I\right)$, when it passes through the PDLM. Each of the $2^{\text {nd }}$ order crosstalk follows a different path and hence has a different delay. Let $d_{i} \tau$ be the delay of the $i^{\text {th }} 2^{\text {nd }}$ order crosstalk, where $\left(0 \leq d_{i} \leqslant\right.$ $2^{n}-1$ ). It can be shown that, by ignoring the higher order terms, the intensity noise caused by the interference between the main signal and the $2^{\text {nd }}$ order inband crosstalk at the output of the PDLM can be expressed as:

$i=0.5\left(E_{i n}\right)^{2} \Sigma\left\{2 b_{s} b_{i} \varepsilon(1-\varepsilon)^{n} \cos \left[\left(d_{s}-d_{i}\right) \omega r+\varphi\left(t-d_{s} \tau\right)-\varphi\right.\right.$ $\left.\left.\left(t-d_{i} \tau\right)\right]\right\}(1 \leq i \leq n)$

where $E_{i n}$ is the input electrical field, $b_{s}$ and $b_{i}$ are the corresponding "mark" or "space" modulation states of the signal and crosstalk, $\omega$ is the carrier frequency and $\varphi(t)$ represents the laser phase fluctuation.

The phase fluctuation of a DFB laser is commonly modeled as Wiener-Levy random process[5-7]. According to the Wiener-Levy model, the DFB laser's phase variation is a nonstationary zero-mean Gaussian random process. However, its phase difference $\Delta \varphi$ between two times is stationary and independent zero-mean Gaussian random process with a variance given by $\sigma_{\Delta \varphi}^{2}=2 \pi \Delta v \Delta \tau$ for a laser with its power spectral density function of Lorentzian line shape, where $\Delta v$ is the $3 \mathrm{~dB}$ linewidth of the laser source and is the time difference[7].

Applying the Wiener-Levy model, the variance of the phase differrence of the beating items in eq. (1) varies from $\sigma_{\Delta \varphi}=2 \pi \Delta v \tau$ to $\sigma_{\Delta \varphi}^{2}=2 \pi \Delta v\left(2^{n}-I\right) \tau$. As an example, for a time slot duration $T=1.5 \mu$ as in the KEOPS project[8], and a laser linewidth of $1 \mathrm{MHz}$, the variance of the phase difference varies from 0.0368 to 9.351 for $n=8$, covering both the coherent and incoherent regimes[5]

We now examine each cosine item in eq. (1). The variance of the phase difference $\Delta \varphi=\varphi\left(t-d_{s} \tau\right)$ $\varphi\left(t-d_{i} \tau\right)$ between the signal and the $i^{t h}$ crosstalk is: $\sigma_{\Delta \varphi}^{2}=2 \pi \Delta v\left|d_{s}-d_{i}\right| \tau$. Let $\varphi_{i}=\left(d_{s}-d_{j}\right) \omega \tau$, the probability density function $f_{i}(y)$ of $y=\cos \left(\varphi_{i}+\Delta \varphi\right)$ is given by eq. (2) [6]:

$f_{i}(y)=\left[2 \pi \sigma_{\Delta \varphi}^{2}\left(1-y^{2}\right)\right]^{-0.5} \times$

$\Sigma\left\{\exp \left[-\left(\cos ^{-1}(y)-\varphi_{i}+2 \pi n\right)^{2} /\left(2 \sigma_{\Delta \varphi}^{2}\right)\right\}+\right.$

$\left.\exp \left[-\left(-\cos ^{-1}(y)-\varphi_{i}+2 \pi n\right)^{2} /\left(2 \sigma_{\Delta \varphi}^{2}\right)\right]\right\}(-\infty<\mathrm{n}<+\infty)$

The mean and variance of each cosine item can be obtained as in eq.(3).

$\mu_{i}=\left\{f_{i}(y) y d y, \sigma_{i}^{2}=b^{2} f_{i}(y) d y-\mu_{2}^{2}(-1 \leq \mathrm{y} \leq 1)\right.$

It is reasonable to assume that each cosine item to be independent because the laser phase noise difference is considered to be a stationary and independent. Hence, according to the central limited theory, with a reasonably large value of $\mathrm{N}$, when the main signal is of "mark' state, the intensity noise in eq. (1) will be a Gaussian distribution with the mean and variance given by:

$\mu_{\text {sum }}=r\left(\Sigma \mu_{i}\right), \sigma_{\text {sum }}^{2}=r^{2}\left(\Sigma \sigma_{i}^{2}\right)(I \leq i \leq N)$

where $r=0.5\left(E_{j n}\right)^{2} \varepsilon(I-\varepsilon)^{n}$. Here the probabilities of the "mark" and "space" states of the crosstalk items are assumed to be equal. In contrast, when the main signal is in the "space" state, the beating item of eq. (1) can be ignored and the noise will mainly be caused by the receiver noise.

The resultant bit error rate (BER) $p_{b e r}\left(d_{s}\right)$ for the main signal delayed by $d_{s} \tau\left(0 \leq d_{s} \leq 2^{n}-I\right)$ can be obtained following the analysis of BER caused by Gaussian noise[9]. Since a packet is likely to experience any of the $2^{\mathrm{n}}$ possible different delays in the PDLM, the average BER can be expressed by eq.(5), assuming an equal probability for each packet delay.

$P_{b e r}=2^{-\mathrm{n}}\left[\Sigma p_{b e r}\left(d_{s}\right)\right]\left(0 \leq d_{s} \leq 2^{n}-I\right)$
4. Numerical Results of BER and Power Penalty

In order to get a better understanding of how the $B E R$ is affected by the $2^{\text {nd }}$ order inband crosstalk the BER and power penalty of the signal are numerically calculated, based on the analysis given above. In the following discussions we have assumed that $\mathrm{BER}=10^{-12}$ when there is no crosstalk (i.e. BER caused by the receiver's noise alone). Fig. 2 shows the numerical results of the BER versus different crosstalk level for various value of $n$. Note that $\Delta v=1 \mathrm{MHz}$, and $\mathrm{T}=1.5 \mu \mathrm{s}$. For a given value of $n$, the BER is significantly increased when the crosstalk level increases from $-30 \mathrm{~dB}$ to $-20 \mathrm{~dB}$, but the BER is almost not affected when the crosstalk level is less than -30 $\mathrm{dB}$. When crosstalk level is greater than $-30 \mathrm{~dB}$, the BER rapidly deteriorates as the value of $n$ increases, because the overall $2^{\text {nd }}$ order inband crosstalk increases in proportion to $n^{2}$. Fig. 3 shows the relation between the BER and the laser linewidth for various values of $n$ with crosstalk level of $-25 \mathrm{~dB}$. This figure clearly shows that, the BER reaches its maximum at a certain laser linewidth. Further increase or decrease in the linewidth will reduce the BER. For a large value of $n$ (e.g. $n=10$ ), a large linewidth will significantly reduce the BER.

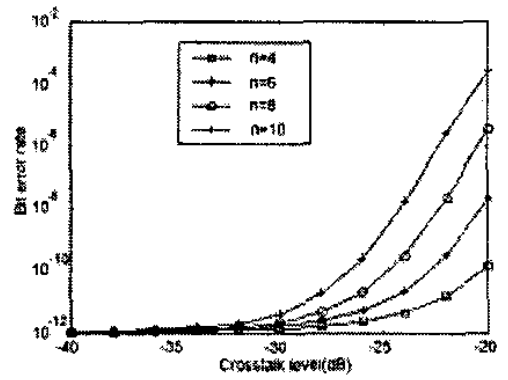

Fig 2 BER vs. crosstalk level for various values of n.

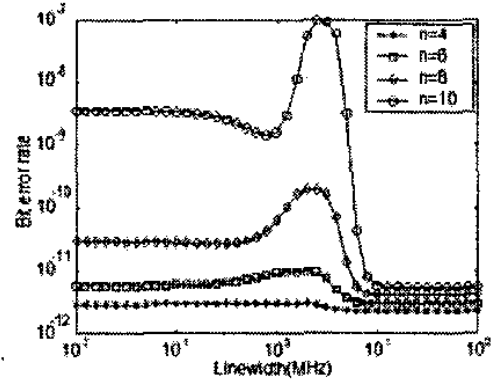

Fig. 3 BER vs. laser linewidth for various values of $\mathbf{n}$.

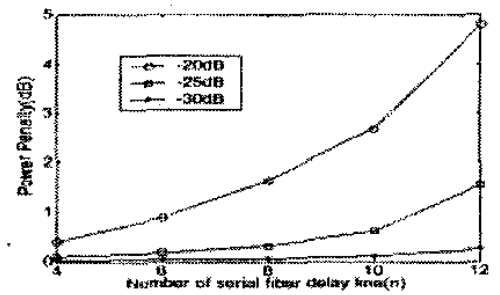

Fig.4 Power penalty versus the value of $n$ for different crosstalk levels

We have also inyestigated the power penalty caused by the $2^{\text {nd }}$ order inband crosstalk. The power penalty is calculated at the BER of $10^{-9}$. power penalty is calculated at the BER of 10
Fig. 4 shows the power penalty versus the value of $n$ for different crosstalk levels. The power penalty is less than $1 \mathrm{~dB}$ when the crosstalk level is less than $-25 \mathrm{~dB}$ and $n \leq 10$. The power penalty is negligible for crosstalk levels less than $-30 \mathrm{~dB}$. However, when the crosstalk approaches to $-20 \mathrm{~dB}$, the power penalty increases rapidly as $n$ increases. A power penalty of $1 \mathrm{~dB}$ only allows for $n=6$ with the crosstalk level of $-20 \mathrm{~dB}$. When the crosstalk level is reduced to $-25 \mathrm{~dB}, n$ is allowed to be increased to 10 for a power penalty of $1 \mathrm{~dB}$.

\section{Conclusion}

We have analyzed the inband crosstalk caused by the synchronization module of photonic packet switches. It has been found that the $2^{\text {nd }}$ order inband crosstalk is dominant but its contribution increases with the number of delay lines, which can cause significantly impact on the BER performance. The numerical study has shown that the BER is significantly affected as the number of delay lines increases. For a reasonable time resolution (i.e. $n>10$ ), the crosstalk level has to be less than $-25 \mathrm{~dB}$ so as to ensure the power penalty is below $\mathrm{I} \mathrm{dB}$.

\section{References}

[1] M. J. O'Mahony, et al, "The Application of optical packet switching in future communication networks," Commun. Mag. 39 128-135, (2001)

[2] R. S. Tucker and W. D. Zhong, "Photonic packet switching: an overview," IEICE Trans. Commu., E82-B, 254-264 (1999)

[3] M. Burzio, et al., "An optical cell synchronizer for packet switched nodes," Proc. Photonics in Switching, pp. 12-17, USA, Mar. 1995

[4] M. P. Earnshaw, et al. "Semiconductor space switches based on multimode interference couplers," J. Lightwave Technol. 20 643-650 (2002)

[5] B. Moslehi, "Analysis of optical phase noise in fiber-optic systems employing a laser source with arbitrary coherence time," $J$. Lightwave Technol. LT-4 1334-1351 (1986)

[6] Arie. A., etal., "Probability-density function of noise at the output of a two beam interferometer," J. Opt. Soc. Am.,A . 1936-1942(1991)

[7] J. L.Gimlett etal., "Effects of phase-to-intensity noise conversion by multiple reflections on Gigabit-per-second DFB laser transmission sys tems," J. Lightwave Technol. 7 888-895(1989)

[8] Guillemot, etal. "Transparent Optical Packet Switching: The European ACTS KEOPS Project Approach," J. Lightwave Technol. 12 2117-2133(1998)

[9] H. Takahashi, etal, "Impact of crosstalk in an arrayed-waveguide multiplexer on $\mathrm{NxN}$ optical interconnection," J. Lightwave Technol. 14 1097-1105, (1996)

FJ6

9:15 AM

Novel Design Method for Generalized Lattice Filters

S. Agger, J. Povlsen, COM - DTU, Copenhagen Lyngby, Denmark; L. Leick, D. Zauner, NKT Integration, Copenhagen - Birkerød, Denmark, Email: as@com.dtu.dk.

A new design method for wide-band gain equalizers using cascaded MZI's is proposed. The method achieves a given accuracy with fewer stages than previously described methods. Furthermore, the method is capable of minimizing PDL.

Introduction

Long haul transmission in the backbone network and functionalities in the metro- and access WDM networks require amplification, which is nommally provided by erbium doped amplifiers. Unfortunately, the spectral gain of an erbium amplifier is non-flat over the $\mathrm{C}$-band, providing different gain at the individual channels, which decreases the channel SNR and eventually leads to errors in the transmission. Gain Equalizing Filters (GEF), having the inverse amplifier transmission, are attractive components for compensating this gain variation. A possible way of realizing a GEF is to cascade N Mach Zehnder Interferometers (MZI) to obtain a transmission, which is an $\mathrm{N}^{\text {th }}$ order Fourier expansion. Previously, the filter parameters were extracted by a recursive method[1] 
However, the method assumes wavelength independent coupling constants and integer delay ratios, which limits the viability. As a consequence, the method is only useable for narrow-band filters. The necessity of including the coupler wavelength dependency for wide-band multiplexers have also been discussed in[2]. In the present paper a new method is proposed that includes coupler wavelength dependency, non-integer delay ratios and a dual fitting scheme that effectively minimizes Polarization Dependent Loss (PDL). It is shown that the new method significantly reduces the approximation error between the desired and the actual transmission. Theory

The basic lattice element in the filter is shown in figure 1 .

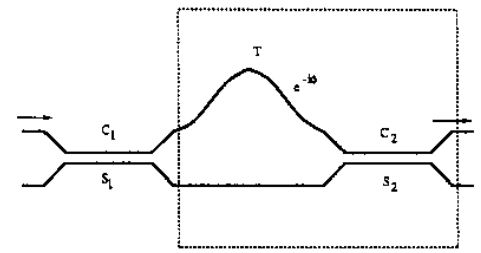

Fig. 1: First order MZI consisting of two couplers and one delay line. The framed part is the basic lattice element.

It consists of a delay line and a directional coupler. The first directional coupler is treated separately and a given filter is realized by cascading basic lattice elements to the first coupler.

The transfer matrix (in field amplitudes) is found by multiplication of the transmission matrices of each individual basic lattice element. The $k^{\prime}$ th transfer matrix is related to the $(\mathrm{k}-\mathrm{I})$ as $[3]$. ,

$$
T^{k}=\left|\begin{array}{cc}
c_{k}\left(\omega_{n}\right) z^{m_{k}} e^{-i \omega_{k}} & -i s_{k}\left(\omega_{n}\right) \\
-i s_{k}\left(\omega_{n}\right) z^{m_{k}} e^{-i \phi_{k}} & c_{k}\left(\omega_{n}\right)
\end{array}\right| T^{k-1}
$$

where $c\left(\omega_{n}\right)$ is the through-coupling constant, $s\left(\omega_{n}\right)$ the cross-coupling constant, $m_{k}$ is the ratio of the $k^{\text {th }}$ lattice elements Free Spectral Range (FSR) and the FSR of the entire filter. The variable $z^{m}=\exp \left(-j m \omega_{n} \tau\right)$ is the harmonic in the normalized frequency $\omega_{\mathrm{n}} \tau=2 \pi\left(\mathrm{f}-\mathrm{f}_{1525 \mathrm{~m}}\right) / \mathrm{FSR}=$ $2 \pi\left(f-f_{1525 \mathrm{~m}}\right) \mathrm{T}$, where $\mathrm{f}$ is the optical frequency, FSR the Free Spectral Range and T=1/FSR is the delay difference between the arms of the interferometer. If $m_{k}>0$, the delay is in the upper arm and if $m_{k}<0$, the delay is in the lower arm. In practice, the phase $\varphi_{k}$ is applied with thermo-optic heaters placed along the longer arm of the interferometer. A non-linear Levenberg-Marquardt algorithm[4]iteratively searches the amplitude of the matrix element $T_{i j}$ corresponding to the input output port of interest for the best fit in a relative least square error sense $\chi^{2}=$ ? $\left(\left|\mathrm{H}\left(\omega_{\mathrm{n}}\right)\right|-\right.$ $\left.\mathrm{T}_{11}\left(\omega_{n} ; \mathrm{a}\right)\right|^{2} /\left|\mathrm{H}\left(\omega_{\mathrm{n}}\right)\right|^{2} \mathrm{~d} \omega$, where $\mathrm{a}$ is a vector of the fitting parameters and $\mathrm{H}\left(\omega_{\mathrm{n}}\right)=\mathrm{G}_{\min } / \mathrm{G}\left(\omega_{\mathrm{n}}\right)$ is the inverse amplifier gain. The fit is made relative to enhance contributions from small values, thereby improving the fit in a logarithmic scale. Notice, that the phase response is not controlled, as the dispersion of wide-band filters is low.

The coupling constants are found by modal analysis in a 3D-BPM program, where the difference in the supermode propagation constant is linearly expanded in the wavelength. To provide a realistic design, the BPM waveguide parameters have been adjusted for best agreement with measurements. The fitting parameters of the design method is the $\mathrm{k}^{\text {th }}$ straight coupling length $\mathrm{L}_{\mathrm{s}, \mathrm{k}}$, the phase shift $\varphi_{\mathrm{k}}$, and the delay ratio $\mathrm{m}_{\mathrm{k}}$.

\section{Linear method vs. Iterative method}

To evaluate the performance of the iterative method, it is compared with a design routine based on linear constant-coefficient Fourier expansion. The linear method is implemented with integer delay ratios and wavelength independent coefficients. Adjusting the phase response in order to improve the amplitude fit optimises the linear least square. Furthermore, the free spectral range is enlarged in an attempt to minimize the error within the bandwidth of the filter. The $2^{\text {nd }}$ and $5^{\text {th }}$ order responses are shown in figure 2 as a function of normalized frequency found by the linear method.

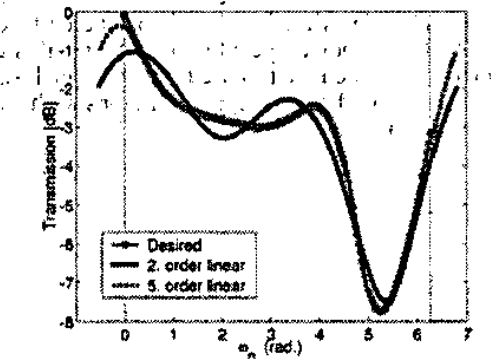

Fig. 2: Filter transmission as a function of normalized frequency for two different filter orders (physical delay number) in the ideal case of constant coefficients. Punctuated lines indicate the $\mathrm{C}$-band. Notice the increase in FSR for improved fit.

The filter was fitted to a measured inverse amplifier response transmission, having a dynamic range of $8 \mathrm{~dB}$ over the whole $\mathrm{C}$-band.

Notice the convergence of the fit as the order increases. The punctuated line indicates the C-band, illustrating the adjustment of the filters FSR. For a direct comparison of the linear and the iterative method, the relative least square enror has been calculated for each method for 5 different lattice numbers and the result is shown in figure 3 .

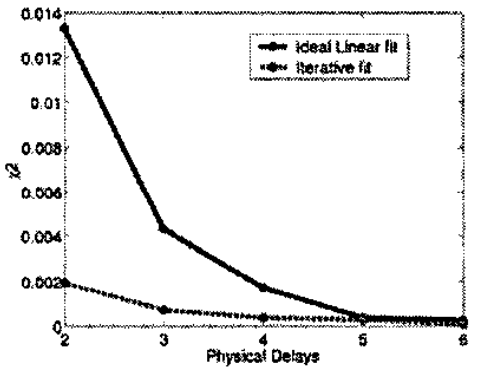

Fig. 3: $\chi^{2}$ as a function of physical delay number lattice number) for the linear- and iterative method. The iterative method shows superior performance due to non-integer delays and couple wavelength dependency. Notice that the ideal linear fit refers to the case of wavelength constant couplers.

The iterative method shows superior performance already at low lattice numbers in comparison to the ideal constant coefficient linear filter. At two delay stages, the iterative method gives error estimate as good as that of a $4^{\text {th }}$ order linear method. For all the considered lattice numbers, the iterative method has an error estimate $\chi^{2}<0.002$, which corresponds to a maximum transmission error of less $0.5 \mathrm{~dB}$ over the entire C-band. The linear method only achieves this for lattice numbers larger than 4 . Including coupler wavelength dependency and non-integer delay ratios is seen to have the effect of enhancing the filter performance. The iterative method shows remarkable improvements, especially for low delay numbers.

\section{Dual fitting scheme}

Yet another interesting ability of the iterative method is to include polarization effects in the fitting process. Assuming a linear system and that the transfer function for the filter is known for two orthogonal polarization eigenstates, then the transfer function of an arbitrary polarization state is given by a linear combination of the two eigenstates $F_{\text {arb pol }}=\alpha F_{\text {eigenstatel }}+(1-\alpha) F_{\text {eigenstate2 }}$, implying that fitting both responses to the same inverse amplifier transfer function will minimize polarization excursions, such as Polarization

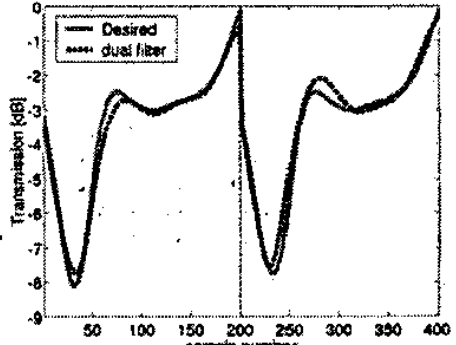

Dependent Loss (PDL). The idea is illustrated in the figure 4

Fig. 4: Principle of the dual fitting scheme. Two different functions representing two orthogonal polarization states fitted to two identical inverse amplifier responses. The fitting routine sees one of the functions in the left half and the other function in the right half.

The desired filter response is artificially duplicated. In one iteration step, the fitting routine is given the response of the first polarization eigenstate, corresponding to the left half in figure and the other polarization eigenstate, corresponding to the right half in figure. The result is a simultaneous least square minimization of both responses, thereby squeezing the responses of arbitrary polarization states.

A scalar 3D-BPM modal analysis with appropriately adjusted waveguide parameters have been applied to obtain the difference in the supermode propagation constants of the coupler for the two polarizations. The waveguide parameters were adjusted to obtain best agreement with polarization measurements.

Birefringence in the delay section introduces a polarization dependent phase shift of magnitude $\Delta \varphi_{b}=k_{c} L \Delta n_{e}$, where $k_{c}$ is the center wavenumber, $\mathrm{L}$ is the relative delay length and $\Delta \mathrm{n}_{\mathrm{e}}$ is the difference in effective indices of the two polarizations. The phase shift is added to the argument of the exponential, which is then given by $z^{\mathrm{m}}=$ $\exp \left(-\mathrm{jm}\left(\omega_{\mathrm{n}} \tau \pm \Delta \varphi_{\mathrm{b}}\right)\right.$. A birefringence of $\Delta \mathrm{n}_{\mathrm{e}}=0.001$ gives a phase shift of $\Delta \varphi_{b}=0.013 \pi$ for $k_{c}=2 \pi$ $1545 \mathrm{~nm}$ and $\mathrm{L}=20.1 \mu \mathrm{m}$ corresponding to a FSR of $40 \mathrm{~nm}$ of the basic lattice elements.

In the figures 5 and 6 , the single - and dual fitting schemes are compared for a $4^{\text {th }}$ order lattice filter with birefringent couplers (PDW of $5.1 \mathrm{~nm} @$ )first crossstate) and $\Delta \varphi_{b}=0.013 \pi$

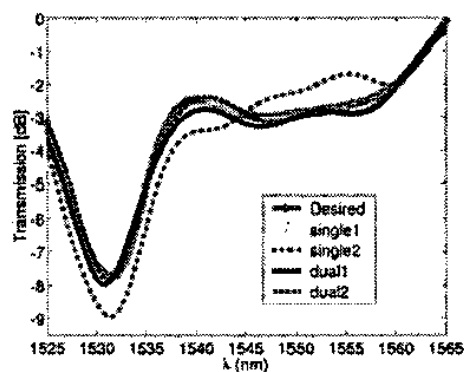

Fig. 5: Transmission of the polarization eigenstates as a function of wavelength for a $4^{\text {th }}$ order lattice filter obtained by the single and dual fitting schemes. The fits are very good, but notice the excursion of one of the single fit transmissions.

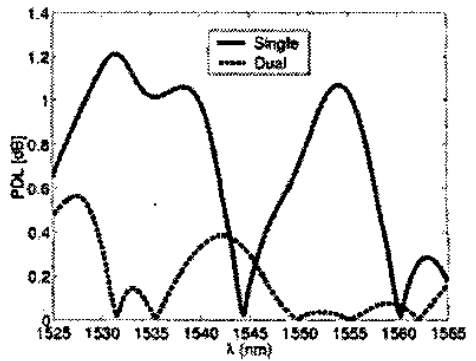


Fig. 6: PDL as a function of wavelength for the dual and single fitting scheme for $4^{\text {th }}$ order lattice filter (see figure 5). The PDL is greatly reduced in the dual scheme.

The single fitting scheme provides an error estimate of $\chi^{2}=6.3 \mathrm{e}-4$ and $\chi^{2}=5.3 \mathrm{e}-2$ for the two polarization transmissions, respectively. The dual scheme provides $\chi^{2}=4.9 \mathrm{e}-3$ and $\chi^{2}=3.30 \mathrm{e}-3$. The dual scheme distributes the error on both transmissions while minimizing their difference to the desired transmission and the effect is evident in the figures, where the PDL of the dual scheme is seen to be much lower. Integrating the PDL over the entire frequency band gives a ratio of the single vs. dual error of 4.16/0.38@11, one order of magnitude better with the dual scheme. This proves the dual scheme schemes applicability of greatly reducing PDL in cascaded MZI filters.

\section{Conclusion}

A novel design method for wide-band filters is presented. It includes coupler wavelength dependency and polarization effects with good performance. In the single fit mode, the method shows improvements of almost two filter orders with respect to common constant-coefficient Fourier expansion methods. In addition, the method has been extended to minimize PDL using a dual scheme fit, which effectively distributes the polarization errors between two orthogonal polarization eigenstate transmissions, while simultaneously fitting to the desired transmission. The dual scheme reduces the PDL by an order of magnitude with respect to the single fitting scheme.

\section{References}

1. K. Jinguji and M. Kawachi, "Synthesis of coherent two-port lattice-form optical delay-line circuit', journal of lightwave technology, vol. 13, pp. 73-82, January 1995

2. C. Henry et al., 'Monolithic optical waveguide filters based on Fourier expansion', United States patent, vol. 5,596,661, Jan. 21, 1997

3. C. K. Madsen and J. H. Zhao, "Optical filter design and analysis: A signal processing approach', chap. 4.5, John Wiley \& Sons, 1999 4. W.H. Press et al., 'Numerical Recipes in C - the ant of scientific computing', ch. 15, Cambridge University Press, 1992

Multi-Channel Chromatic Dispersion Compensator Consisting of Modified Interleave Filter

M. Oguma, K. Takiguchi, T. Kitoh, S. Suzuki, T. Shibata, T. Mizuno, NTT Photonics Laboratories, Atsugi-shi, Japan, Email: oguma@aecl.ntt.co.jp.

We have successfully demonstrated a low loss dispersion slope compensator consisting of a PLC-type modified interleave filter. It has different chromatic dispersions to meet the demand of each $50 \mathrm{GHz}$-spacing WDM channel in the $C$-band.

\section{Introduction}

The dispersion compensation of transmission fiber is indispensable in high-bit-rate (e.g. $40 \mathrm{~Gb} /$ s) wavelength division multiplexing (WDM) transmission systems. Of the various possible approaches, distributed fiber management is one of most likely candidates, especially for long-hau systems. With this method, we can easily design the chromatic dispersion so that it is zero at a given wavelength. However, it is still difficult to compensate completely for the dispersion differentials between the WDM channels over a wide wavelength range. Numerous devices have been developed to remove these residual dispersions, which are mainly due to the dispersion slope of optical fiber. These devices include arrayed tunable or preset compensators [1-3] and multi-channel compensators with a channel selector $[4,5]$ Recently another method has been reported that employs a pair of filters with opposite dispersion slopes indicating the possibility of compensating for dispersions in multi channels simultaneously
[6]. However, the filters are composed of bulk optics, mirrors and a circulator, giving them complicated structure and a relatively high insertion loss.

In this paper, we demonstrate a compact and low-loss dispersion compensator for DWDM systems fabricated by using planar lightwave circuits (PLC). The device offers the advantages of dense integration, precise control of the filter characteristics, mass-producibility and long-term stability The fabricated device was based on a lattice-form interleave filter and it achieved a different chromatic dispersion at each $50-\mathrm{GHz}$ spaced WDM channel from 1530 to $1565 \mathrm{~nm}$, which forresponds to a dispersion slope of $-5.7 \mathrm{ps} / \mathrm{nm}^{2}$. We also achieved a low insertion loss of $2.5 \mathrm{~dB}$ in the C-band.

\section{Operating principle and basic design}

Here we describe the basic principle we used for designing the proposed dispersion slope compensator. Figure 1(a) shows the schematic configuration of the fundamental unit that we used for constructing the compensator and Fig. 1(b) shows its simulated transmittance and relative group-delay responses.

The compensator consists of three of these fundamental units with different center wavelengths, as described below. The fundamental unit is composed of a lattice-form filter, and is almost the same as that previously reported as an interleave filter [7]. The unit is designed to have a rectangular transmittance and a parabolic group-delay response simultaneously. This type of filter generally has a special feature in that we can choose its opposite group-delay response by selecting it input and output ports as shown in Fig. 1(b). By using this feature, an interleave filter with no chromatic dispersion has been demonstrated by cascading the fundamental units [8].

Moreover, it is possible to control the chromatic dispersion of the multi-stage filter by shifting the center frequency of the units with opposite filter responses [6]. Therefore, we constnucted a multi-channel dispersion compensator by cascading the filter units as shown in Fig. 2 (a). We used two kinds of unit (filters 1 and 2 in Fig. 2), which had slightly different channel spacings (a difference of $2 \delta$ between the two spacings) and whose group-delay responses had opposite convex directions. The filter configuration described in Fig. 2 (a) enabled us to change the chromatic dispersion gradually at each channel as shown Fig. 2 (b). The dispersion slope of total circuit $\beta$, which is proportional to that of unit $\alpha$, is given by the following equation.

$$
\beta=4 \alpha \delta / \Delta \lambda
$$

Here $\Delta \lambda$ is the channel spacing. With this equation, we can easily design the dispersion slope over a wide wavelength range, but as $\delta$ becomes larger, the transmission bandwidth of the outer channels in a high channel count WDM system becomes narrower. We therefore chose $50 \mathrm{GH}$ and $0.2 \mathrm{GHz}$ as the $\Delta \lambda$ and $\delta$ values to realize a narrow channel spacing operation throughout the $\mathrm{C}$-band. The dispersion slope of the fundamental unit was approximately $+/-350 \mathrm{ps} / \mathrm{nm}^{2}$. These values corresponded to the compensation of the dispersion slope in an $80 \mathrm{~km}$-long dispersion shifted fiber (DSF), namely, $-5.7 \mathrm{ps} / \mathrm{nm}^{2}$ in the C band.

\section{Experimental results}

We fabricated the dispersion compensator by using a conventional $\mathrm{GeO}_{2}$-doped silica-based waveguide on a Si-substrate. The waveguide had a relative index difference of $0.75 \%$ between the core and the cladding and a core size of $6 \times 6 \mu \mathrm{m}^{2}$ Each coupler in the filter was composed of three-stage Mach-Zehnder interferometers to stabilize its coupling ratio. The total waveguide length of the cascaded filter was more than 200 $\mathrm{mm}$. To reduce the chip size, we used a folded waveguide layout for the cascaded lattice-form

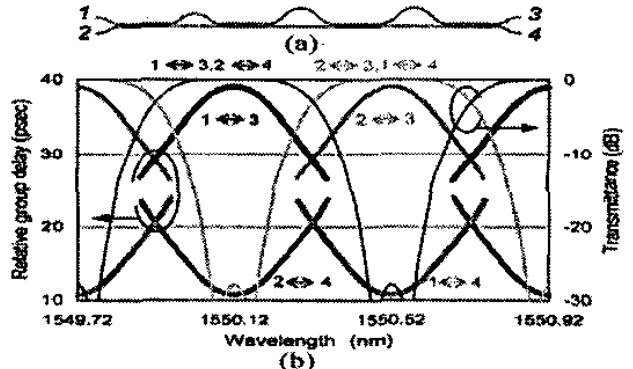

Fig.1 (a) Schematic configuration of a fundamental filter unit. (b) Simulated transmittances and delay time responses for each set of input and output ports in the filter unit.
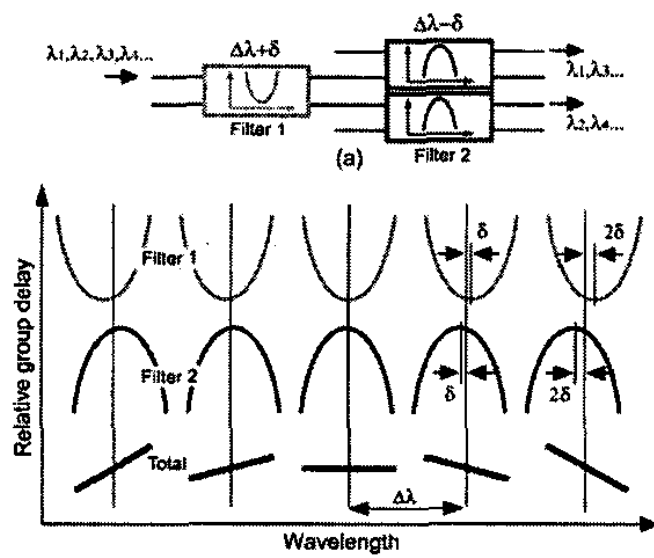

(b)

Fig.2 Operating principle of our chromatic dispersion slope compensator. (a) Device configuration consisting of two kinds of fundamental unit. (b) Relative group-delay response of fundamental units with different convex directions and channel spacing. 